\title{
uniculus paca Linnaeus, 1766 (Cuniculidae, Rodentia) no Holoceno da Gruta Cuvieri, Brasil
}

\author{
Artur Chahud \\ Universidade de São Paulo, Instituto de Biociências, Laboratório de Estudos Evolutivos \\ Humanos, Rua do Matão, 277, 05.508-090, São Paulo, Brasil, arturchahud@yahoo.com
}

\begin{abstract}
Resumo: A região de Lagoa Santa é conhecida pelo importante complexo de cavernas, sendo que em muitas delas encontra-se material osteológico de fauna extinta e atual. A Gruta Cuvieri faz parte deste importante complexo cárstico e possui grande quantidade de restos de roedores do Quaternário, sendo que a família Cuniculidae é a que apresentou os maiores espécimes. O presente estudo comenta os restos de Cuniculus paca de idade holocênica da Gruta Cuvieri. Os restos são do Holoceno Médio e Final e incluem três indivíduos adultos desarticulados e incompletos. O aparecimento desta espécie ocorre simultaneamente com o aumento de umidade na região de Lagoa Santa. Ocorrências dessa espécie em sítios arqueológicos e paleontológicos de idade holocênica são comuns, mas pouco divulgadas, e a publicação dessas ocorrências poderá contribuir futuramente para estudos evolutivos de Cuniculidae no território brasileiro.
\end{abstract}

Palavras-chave: Cavioidea, Quaternário, América do Sul, Lagoa Santa.

\section{Cuniculus paca Linnaeus, 1766 (Cuniculidae, Rodentia) in the Holocene of the Cuvieri Cave, Brazil}

Abstract: The region of Lagoa Santa is known for its important complex of caves, many of which contain osteological material from extinct and current fauna. The Cuvieri Cave is part of this important karst complex and has a large amount of Quaternary rodent remains, with the Cuniculidae family presenting the largest specimens. The present study comments on the remains of Cuniculus paca of Holocene age from the Cuvieri Cave. The remains are from the Middle and Late Holocene and include three disarticulated and incomplete adult individuals. The appearance of this species occurs simultaneously with the increase in humidity in the Lagoa Santa. Occurrences of this species in archaeological and paleontological sites of Holocene age are common, but poorly known, and the publication of these occurrences may contribute to evolutionary studies of Cuniculidae in the Brazilian territory in the future.

Keywords: Cavioidea, Quaternary, South America, Lagoa Santa.

\section{INTRODUÇÃo}

Roedores da Superfamília Cavioidea Fischer, 1817 são exclusivos das Américas e nela estão incluídas as capivaras, cutias, cutiaras, maras, mocós, pacas, preás e porquinhos-da-
Índia, totalizando mais de 40 espécies (Mammal Diversity Database, 2021).

As Pacas (gênero Cuniculus Brisson, 1762) são as únicas representantes da família Cuniculidae, sendo composta por duas espécies atuais; Cuniculus paca (Linnaeus, 1766) e Cuniculus taczanowskii (Stolzmann, 1865). A espécie mais 
comum desta família é C. paca, que ocorre desde o sul do México, passando pela América Central, até o norte da Argentina, sul do Brasil e norte do Uruguai na América do Sul (Emmons, 2016; Leuchtenberger et al., 2016).

Fósseis da família Cuniculidae são pouco conhecidos, sendo a mais antiga ocorrência baseada em um astrágalo descrito por Bergqvist et al. (1998) do Neógeno do Acre, porém este é um espécime considerado indeterminado (Kerber, 2017). Atualmente se reconhece uma única espécie extinta atribuída a esta família, Cuniculus rugiceps (Lund, 1837), da região tropical do território brasileiro, com registros no no final do Pleistoceno (Mayer et al., 2016). A espécie foi identificada primeiramente por Peter Wilhelm Lund na região de Lagoa Santa, Estado de Minas Gerais e posteriormente revisada por Mayer et al. (2016).

A Gruta Cuvieri foi alvo de inúmeros trabalhos de datação, paleoambientais (Hubbe et al., 2011), paleontológicos (Alvarenga et al., 2008; Mayer et al., 2016; 2020; Chahud, 2020a; 2020b; 2020c; 2021; Chahud \& Okumura, 2021a; 2021b; 2022; Chahud et al., 2020), espeleológicos e sedimentológicos (Haddad-Martim et al., 2017). Recentemente, Chahud (2020a) estudou a presença de C. paca do Pleistoceno da Gruta Cuvieri, mas nenhum trabalho detalhou a ocorrência em depósitos mais recentes nesta caverna.

Observando os poucos trabalhos envolvendo pacas atuais em sítios paleontológicos e arqueológicos, a presente contribuição tem por objetivo apresentar os espécimes de Cuniculus paca do Holoceno da Gruta Cuvieri, além de breves comentários sobre a espécie no registro fóssil e arqueológico em território brasileiro.

\section{MATERIAL e MÉtodos}

A Gruta Cuvieri é constituída de três pequenas cavidades verticais, armadilhas naturais, que foram denominadas Locus 1, 2 e 3 (Fig. 1), com 16 metros, 4 metros e 8 metros de profundidade respectivamente. Todo o material estudado nesta contribuição é proveniente do Locus 2, de idade predominantemente holocênica (Hubbe et al., 2011; Mayer et al., 2016; Haddad-Martim et al., 2017). Este depósito é pouco consolidado em sua maior parte, o que permitiu a execução
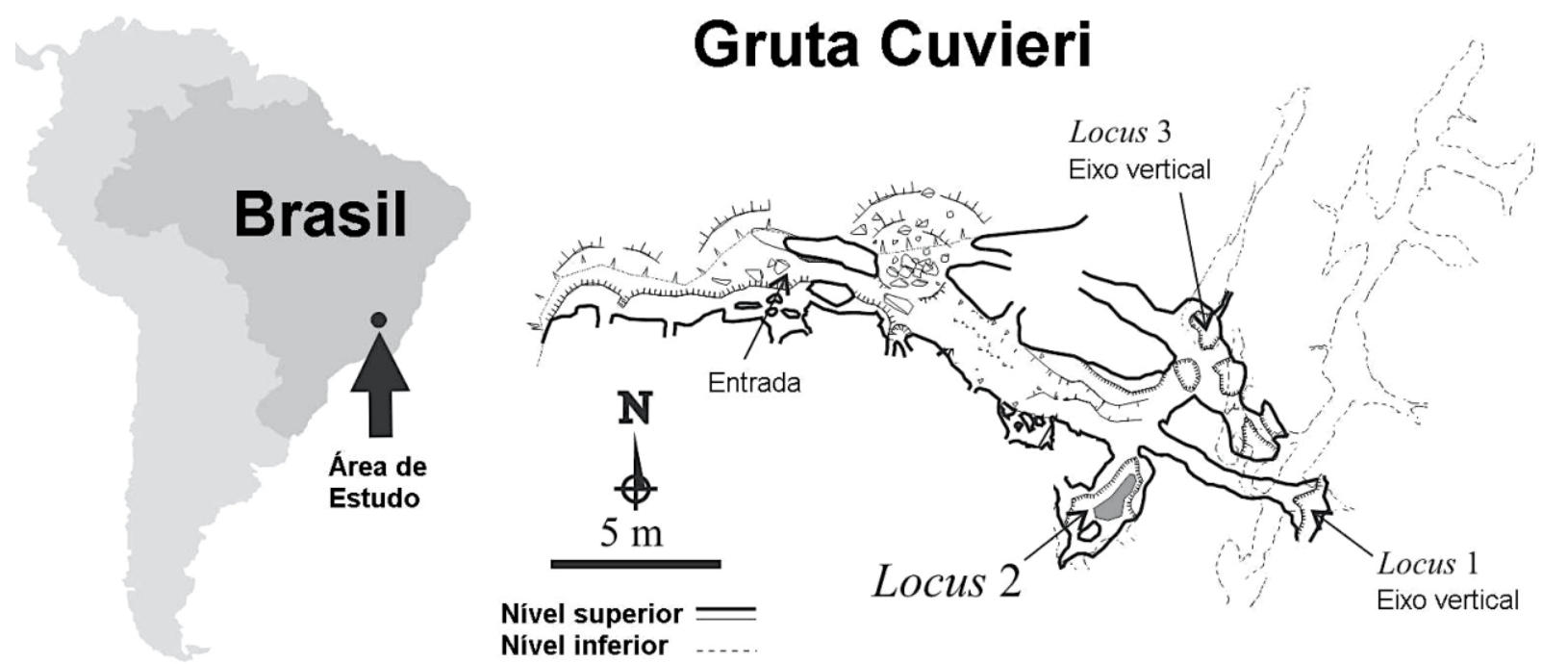

Fig. 1. Localização geográfica da área de estudo e da Gruta Cuvieri mostrando a posição do Loci 1, 2 e 3 (mapa cortesia de Alex Hubbe e Grupo Bambuí de Pesquisas Espeleológicas).

Fig. 1. Geographic location of the study area and of Cuvieri Cave showing the position of Loci 1,2 and 3 (map courtesy of Alex Hubbe and Grupo Bambuí for Speleological Research).

de escavações estratigraficamente detalhadas, que registrou e posicionou em esquemas e croquis detalhados a posição de cada parte óssea nos depósitos do Locus 2.

Os aspectos tafonômicos como abrasão ou desgaste ósseo, nível de exposição a intempéries, quebras, atividade biológica (carniceiros ou predadores) e presença de articulação foram os itens avaliados seguindo os conceitos de Voorhies (1969), Badgley (1986), Behrensmeyer 
(1978; 1991) e Lyman (1994). Para a identificação dos espécimes foi feita a comparação com os exemplares conhecidos da Coleção Renato Kipnis e da Coleção Etnográfica Guajá do Laboratório de Estudos Evolutivos Humanos do IB-USP, que possui material osteológico atual, além de consulta às obras de Paula Couto (1979), Chahud (2019), Ríos-Uzeda et al. (2004), Woods \& Kilpatrick (2005), Castro et al. (2010), MendesOliveira et al. (2012), Mayer et al. (2016), Kerber, (2017) e Hingst-Zaher \& Brandão (2021).

Os espécimes da Gruta Cuvieri citados nesse trabalho e o inventário de suas partes ósseas estão depositados no Laboratório de Estudos Evolutivos Humanos (LEEH) do Instituto de Biociências da Universidade de São Paulo (IBUSP).

\section{Caracterização da Área de Estudo}

O Locus 2 da Gruta Cuvieri é caracterizado por duas fácies sedimentares distintas de coloração e grau de cimentação diferenciados, mas sem estrutura sedimentar visível. O contato é considerado discordante sendo que a fácies inferior é diferenciada da superior por uma coloração marrom avermelhada mais escurecida e maior cimentação, se comparada com a fácies superior (Hubbe et al., 2011), de coloração marrom escuro.

O depósito do Locus 2 foi pouco remobilizado e manteve a maior parte da estratigrafia, sendo possível constatar que o material osteológico encontrado em camadas mais profundas possui idades mais antigas e próximo da superfície, mais recentes (Hubbe et al., 2011).

Baseada na datação do material osteológico a idade da fácies inferior é considerada como limite entre o Pleistoceno-Holoceno, enquanto a superior pertence ao Holoceno (Hubbe et al., 2011).

O material reportado no presente estudo é exclusivo de porções intermediárias da camada superior e, ao menos três indivíduos puderam ser diferenciados, sendo que dois foram datados por Hubbe et al. (2011).

O espécime mais antigo, CVL2-5998, obteve idade convencional 14C AMS de 5050 \pm 40 antes do presente (AP), calibrada $2 \sigma$ cal entre 5910 e 5670 AP (Hubbe et al., 2011). Este espécime datado do fim do Holoceno Médio está entre as peças ósseas encontradas em profundidades maiores $(-0,80 \mathrm{~m}$ abaixo do datum), sendo, provavelmente, a mais antiga no Locus 2.

O espécime CVL2-P258/234 é de idade recente, 220 \pm 40 AP (Hubbe et al., 2011) e foi encontrado próximo da superfície, nas primeiras exposições de escavação $(-0,62 \mathrm{~m}$ abaixo do datum). O outro indivíduo aparentemente deva ter idade similares, pois seus restos também foram observados nos mesmos níveis deste espécime.
Atualmente a ocorrência de pacas na região de Lagoa Santa foi reportada por Trolle et al. (2006) que a partir de entrevistas com fazendeiros, registraram a presença da espécie.

\section{Resultados e Discussão}

Ordem Rodentia Bowdich, 1821

Infraordem Hystricognathi Tullberg, 1899

Superfamília Cavioidea Fischer, 1817

Família Cuniculidae Miller \& Gidley, 1918

Gênero Cuniculus Brisson, 1762

Cuniculus paca Linnaeus, 1766

Material: o material osteológico analisado inclui; uma ulna esquerda (CVL2-462), dois úmeros direitos (CVL2 463A-463B (epífise), e CVL2 2635), um astrágalo esquerdo (CVL2 P1883), um astrágalo direito (CVL2 4761), um úmero esquerdo (CVL2 3634), um calcâneo esquerdo (CVL2 446), um calcâneo direito (CVL2 3354), dois dentários direitos (CVL2 351-352 e CVL2 444A), parte de arco zigomático (CVL2 407A), um fêmur esquerdo fragmentado (CVL2 6486), três epífises distais de fêmur esquerdo (CVL2 6357 e CVL2 601 e CVL2 760) e uma epífise distal de fêmur direito (CVL2 4627) (Fig. 2).

Comentários gerais: 0 material recuperado de C. paca é constituído de fragmentos de dois dentários de indivíduos diferentes, mas encontrados em posições estratigráficas similares. Ambos possuem dentição de pré-molares e molares de animais adultos (Chahud, 2019; HingstZaher \& Brandão, 2021). A idade de pelo menos um dos espécimes como adulto é confirmado pelo arco zigomático muito rugoso, comparável ao observado em espécimes adultos atuais e pleistocênicos(Mayer et al., 2016; Chahud, 2019; 2020; Hingst-Zaher \& Brandão, 2021).

Um dos úmeros teve a epífise proximal não totalmente fusionada, sugerindo que não fosse um animal adulto idoso. Os outros ossos são típicos de indivíduos adultos atuais, comparáveis em tamanho e forma com espécimes estudados por Chahud (2019) da região da Amazônia do Estado do Maranhão.

Caracterização tafonômica: Os depósitos da Gruta Cuvieri serviram como armadilha para os macrovertebrados que lá entravam e acabavam presos em seus interiores. Isso foi observado em ungulados (Hubbe et al., 2011; Chahud, 2020c; Chahud \& Okumura, 2021b; Mayer et al., 2020), grandes felinos (Chahud, 2020b; Chahud \& Okumura, 2021a), coelhos (Chahud et al., 2020) e para roedores do Locus 3 (Mayer et al., 2016; Chahud, 2020a).

Os restos osteológicos de ungulados (Tayassuidae, Cervidae e Tapiridae) encontrados no Locus 2 são representados por restos articulados, semi-articuladas ou com ossos desarticulados, porém associados a um mesmo indivíduo, além de alguns ossos isolados (Hubbe et al., 2011; 


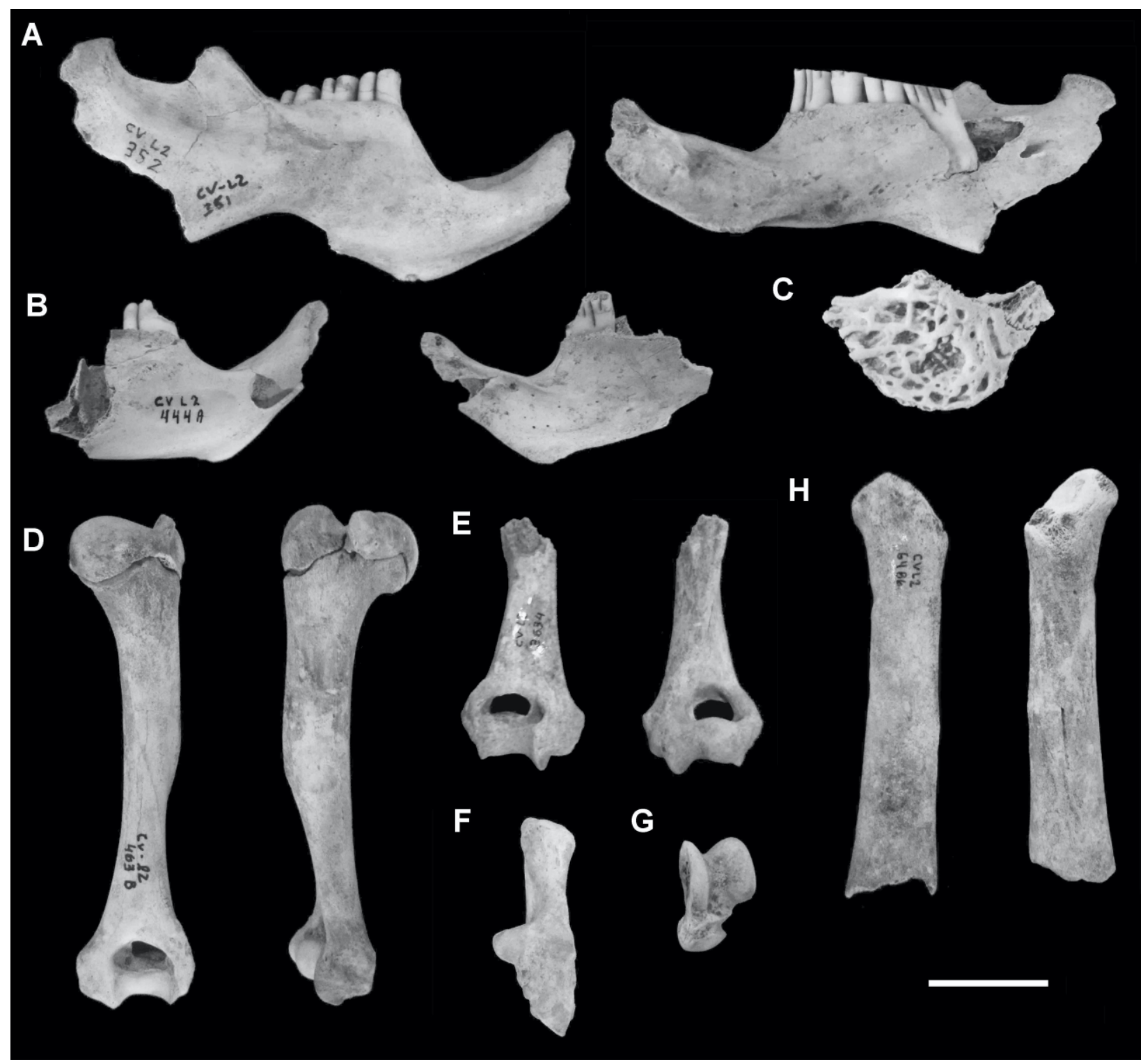

Fig. 2. Material osteológico de Cuniculus paca (Linnaeus, 1766) recuperado no Locus 2. A. Dentário direito incompleto (CVL2 351 e 352). B. Dentário direito fragmentado (CVL2 444A). C. Parte de arco zigomático (CVL2 407A). D. Duas vistas do úmero direito (CVL2 463A-463B). E. Duas vistas do úmero esquerdo (CVL2 3634). F. Calcâneo esquerdo (CVL2 446). G. Astrágalo direito (CVL2 4761) e H. Fêmur esquerdo fragmentado (CVL2 6486). Barra: 20mm.

Fig. 2. Osteological material of Cuniculus paca (Linnaeus, 1766) recovered at Locus 2. A. Incomplete right tooth (CVL2 351 and 352). B. Fragmented right tooth (CVL2 444A). C. Part of the zygomatic arch (CVL2 407A). D. Two views of the right humerus (CVL2 463A-463B). E. Two views of the left humerus (CVL2 3634). F. Left calcaneus (CVL2 446), G) right astragalus (CVL2 4761) and H. Fragmented left femur (CVL2 6486). Bar: 20mm.

Chahud, 2020c; Chahud \& Okumura, 2021b).

Os espécimes de $C$. paca estão todos desarticulados e dispersos, mas em níveis similares (sugestivo de espalhamento e dispersão horizontal). A maior parte dos ossos possui pouco ou nenhum desgaste e apenas um fêmur (Fig. 2H) apresentou quebras e polimento das extremidades. A evidência de alguma remobilização e retrabalhamento são constatadas com a ausência de ossos muito pequenos ou frágeis, estes que seriam os primeiros a serem destruídos ou perdidos em ambientes com algum transporte (Voorhies, 1969). 


\section{Registros de Cuniculidae}

Os registros osteológicos e fossilíferos dessa família são escassos em trabalhos científicos, porém não são incomuns em depósitos quaternários. A primeira identificação foi feita por Lund na região de Lagoa Santa (Lund, 1837; Mayer et al., 2016), com duas espécies; C. paca e C. rugiceps.

A espécie $C$. rugiceps é a única extinta da família e foi, recentemente, revisada taxonomicamente por Mayer et al. (2016) que consideraram a principal característica o tamanho muito maior que a espécie atual. Mayer et al. (op. cit.) comentaram que a espécie extinta teria idades próximas de 29 mil anos para os exemplares da Gruta Cuvieri. Posteriormente, Chahud (2020a) observou que associados a exemplares desta espécie ocorrem indivíduos da espécie recente $C$. paca no Locus 3 (Fig. 3), sugerindo que ambas teriam vivido durante o Pleistoceno, mas ressalta que, diferente do Locus 2, os depositos do Locus 3 tiveram grande remobilização do material osteológico e que, por isso, as espécies não necessariamente foram simpátricas.

Atualmente $C$. paca ocorre em todo o território brasileiro e é compreensível que ocorra em depósitos do Quaternário de diversos estados, como Minas Gerais, Bahia, Pará, Mato Grosso do Sul, Goiás, Paraná, Piauí e São Paulo (Paula Couto, 1979; Mayer et al., 2016; Kerber, 2017; Chahud, 2021).

O material osteológico de C. paca encontrado no Estado de São Paulo esteve entre os mais estudados e foi descrito inicialmente por Chahud (2005) no Abismo Ponta de Flecha, que atribuiu idade recente. Os espécimes se apresentavam muito fragmentados e com os ossos apendiculares com as extremidades ausentes (Chahud, 2021).
Restos de C. paca são muito comuns em material descartado de comunidades indígenas e sítios arqueológicos do Quaternário sul-americano (Chahud, 2019; 2021; Bandeira et al., 2016, Chahud et al., 2021), com grande quantidade de material identificável, porém poucos foram os trabalhos que detalharam estes registros. Em material oriundo de descarte da comunidade Awá-Guajá, do Estado do Maranhão, a maior parte dos roedores coletados e consumidos pela comunidade pertencia a indivíduos de C. paca, sendo em número maior que os representantes de cutias e roedores menores (Chahud, 2019).

O consumo deste animal também foi comum em sítios arqueológicos, mas o registro não é abundante ou foi considerado menos importante por diversos pesquisadores, e normalmente este material não é figurado, com apenas citações da presença da espécie nestes sítios (Plens, 2010; Mingatos \& Okumura, 2016).

Os sambaquis da região sul e sudeste do Brasil apresentaram restos de pacas, normalmente representado por poucos ossos e fragmentos (Figuti, 1993; Pavei et al., 2015). Os sambaquis da região nordeste do Maranhão possui o registro de $C$. paca, como identificado por Bandeira et al. (2016) no Sambaqui Bacanga.

A ocorrência de $C$. paca está presente em sambaquis fluviais, cujos restos foram observados em sambaquis do Vale do Ribeira em São Paulo (Plens, 2010).

Em outros sítios arqueológicos a ocorrência não é mais abundante do que as observadas em sambaquis. No entanto é comum, como observado na Lapa do Santo na região de Lagoa Santa, porém neste caso foram observados espécimes em camadas do início do Holoceno (Mingatos \& Okumura, 2016; Chahud et al., 2021).

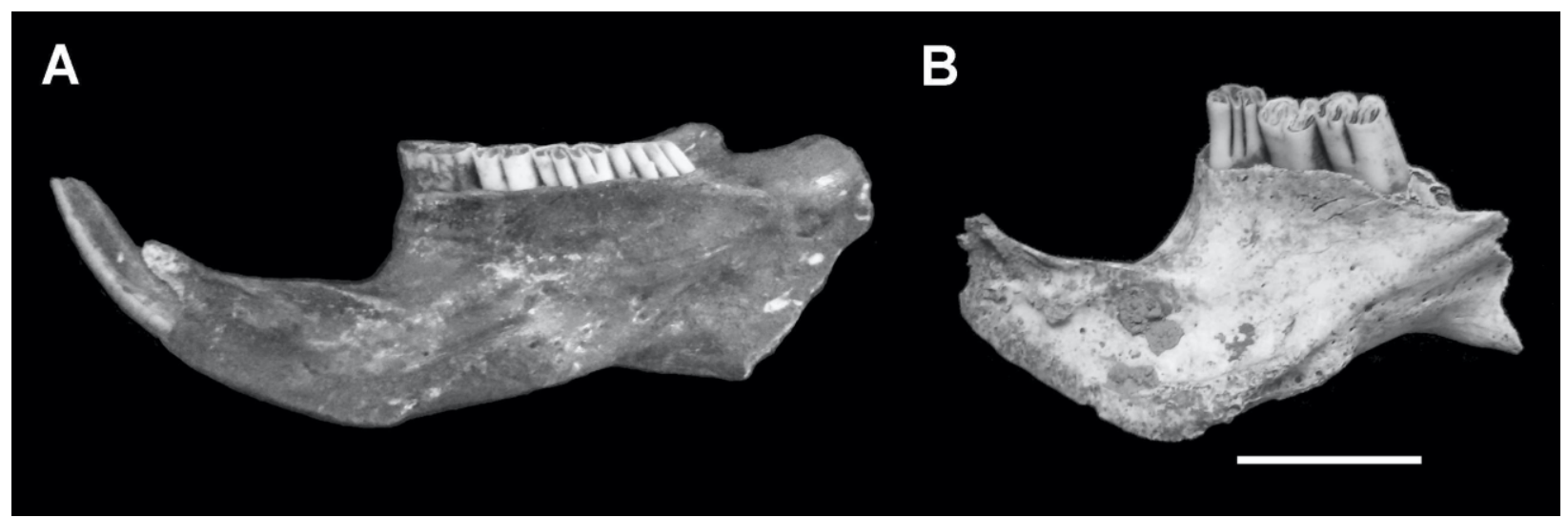

Fig. 3. Dentários de espécimes de Cuniculus do Locus 3 da Gruta Cuvieri. A e B. Cuniculus paca (Linnaeus, 1766) (CVL3-P15403 e CVL3-3790). Barra: 20mm.

Fig. 3. Dentaries of Cuniculus specimens from Locus 3 of the Cuvieri Cave. A and B. Cuniculus paca (Linnaeus, 1766) (CVL3-P15403 and CVL3-3790). Bar: 20mm. 


\section{Considerações Paleoambientais}

C. paca não possui registro na Gruta Cuvieri em idades anteriores a 6000 AP e nesse período as condições ambientais no Holoceno Médio, aproximadamente entre 8000 e 6200 anos (Araujo et al., 2005; 2006; Barros et al., 2011), eram mais secas que as atuais e pode ter sido uma época que não era favorável a presença desta espécie. As condições de aumento da umidade teriam sido intensificadas a partir de 5400 AP e após 4600 AP as condições seriam próximas das atuais (Parizzi et al., 1998).

A presença de C. paca na região de Lagoa Santa no inicio do Holoceno foi registrada em material proveniente da Lapa do Santo (Mingatos \& Okumura, 2016) e pode ter ocorrido durante a primeira fase úmida do Holoceno (Araujo et al., 2005; 2006). Infelizmente as ocorrências do Holoceno Médio em outros sítios paleontológicos e arqueológicos da Lagoa Santa não possuem datação, porém é sugestivo, a partir das datações da Gruta Cuvieri, que houve uma redução, ou ausência, durante o período entre 8000 e 6200 anos de clima mais seco na região, voltando a ocorrer na região a partir de $5400 \mathrm{AP}$, quando o clima apresentou uma nova fase úmida.

\section{Considerações Finais}

Os espécimes coletados no Locus 2 da Gruta Cuvieri são indistinguíveis de espécimes atuais de Cuniculus paca. A espécie também já foi observada em trabalhos anteriores durante o Pleistoceno Final (Kerber, 2017), incluindo a associação de restos com animais extintos da megafauna (Chahud, 2005; 2020a).

Entre os três exemplares identificados, dois são encontrados em épocas recentes, sendo que um foi datado com 220 $440 \mathrm{AP}$, enquanto o terceiro foi datado com $5050 \pm 40$ AP (Hubbe et al., 2011). A ocorrência em períodos recentes não exclui a possibilidade de ocorrer em períodos mais antigos, pois o número de exemplares encontrados é muito pequeno, porém a ocorrência de dois indivíduos em idades recentes e a presença atual da espécie na região sugere uma abundância maior no final do Holoceno ou um retorno desta espécie, considerando a não existência durante parte do Holoceno médio na região.

\section{Agradecimentos}

O autor agradece a CNPq pela bolsa de pós-doc sênior, processo número: 103934/20200 . Agradecimento especial à Professora Doutora Maria Mercedes Martinez Okumura responsável pelo Laboratório de Estudos Evolutivos Humanos do Instituto de Biociências, onde a coleção osteológica Guajá está depositada e aos pesquisadores que coletaram o material da Gruta Cuvieri e que forneceram informações importantes para a realização deste trabalho.

\section{REFERÊNCIAS}

Alvarenga, H., G. R., R. Brito, R. Migotto, A. Hubbe, \& E. Höfling. 2008. Pleistovultur nevesi gen. et sp. nov. (Aves: Vulturidae) and the diversity of condors and vultures in the South American Pleistocene. Ameghiniana. 45(3): 613-618.

Araujo, A. G. M., W. A. Neves, L. Pilo \& J. P. Atui. 2005. Holocene dryness and human occupation in Brazil during the "Archaic Gap". Quat. Res. 64: 298-307. DOI: https:// doi.org/10.1016/j.yqres.2005.08.002

Araujo, A. G. D. M., L. B. Piló, W. A. Neves \& J. P. V. Atui. 2006. Human occupation and paleoenvironments in South America: expanding the notion of an "Archaic Gap". Rev. Mus. Arqueol. Etnol. 15-16: 3-35. DOI: https://doi.org/10.11606/issn.24481750.revmae. 2006.89707

Bandeira, A. M., A. Chahud, I. C. P. Ferreira \& M. L. A. F. Pacheco. 2016. Mobilidade, subsistência e apropriação do ambiente: contribuições da zooarqueologia sobre o sambaqui do Bacanga, São Luís, Maranhão. Bol. Mus. Para. Goeldi. Ciências Humanas. 11(2): 467-480. DOI: https:// doi.org/10.1590/1981-81222016000200007

Badgley, C. 1986. Counting individuals in mammalian fossil assemblages from fluvial environments. Palaios. 1(3): 328-338.

Barros L, C. Lavarini, L. Lima \& A. Júnior. 2011. Synthesis of the Late-Quaternary palaeobioclimatic scenarios in Minas Gerais State/Southeastern Brazil. Soc. Nat. 23: 371-386.

Behrensmeyer, A. K. 1978. Taphonomic and ecologic information from bone weathering. Paleobiology. 4(2): 150-162.

Behrensmeyer, A. K. 1991. Terrestrial Vertebrate Accumulations. Taphonomy. pp. 291335. In: Allison, P.A. \& D. E. G. Briggs (Eds.). Taphonomy: releasing the data locked in the fossil record. New York, Plenum Press.

Bergqvist, L. P., A. M. Ribeiro \& J. Bocquentin-Villanueva. 1998. Primata, Roedores e Litopternas do Mio-Plioceno da Amazônia Sul-Ocidental (Formação Solimões, Bacia do Acre), Brasil. Geolog. Colomb. 23: 19-29. 
Castro, J. J., J. B. López \& F. Becerra. 2010. Una nueva especie de Cuniculus (Rodentia: Cuniculidae) de la cordillera Central de Colombia. Revista ACCB. 22: 122-131.

Chahud, A. 2005. Paleomastozoologia do Abismo Ponta de Flecha, Iporanga, SP. pp. 7678. In: II Congresso Latino-Americano de Paleontologia de Vertebrados. Boletim de Resumos. Rio de Janeiro: Museu Nacional/ UFRJ, Paleo. Dest.

Chahud, A. 2019. Uma coleção osteológica de roedores derivada de atividades de caça da Sociedade Awá-Guajá do estado do Maranhão. Acta Biol. Catar. 6: 83-94.

Chahud, A. 2020a. Dasyproctidae e Cuniculidae (Cavioidea, Rodentia) do Pleistoceno da Gruta Cuvieri, Estado de Minas Gerais, Brasil. BIOTERRA. 20(1): 29-37.

Chahud, A. 2020b. Occurrence of the sabretooth cat Smilodon (Felidae, Machairodontinae) in the Cuvieri cave, eastern Brazil. Palaeontologia Electronica 23(2): a24. DOI: https://doi.org/10.26879/1056

Chahud, A. 2020c. Um exemplar muito jovem de Mazama sp. encontrado na Gruta Cuvieri, Região de Lagoa Santa, Estado de Minas Gerais, Brasil. Rev. Bras. Zoociências. 21(1): 1-10. DOI: https://doi.org/ 10.34019/2596-3325.2020.v21.29276

Chahud, A. 2021. Grandes roedores do Abismo Ponta de Flecha (Quaternário), Iporanga, Brasil. Acta Biol. Paranaense. 50(1-4): 93102. DOI: http://dx.doi.org/10.5380/ abp.v50i1-4.82998

Chahud, A., G. F. Figueiredo, G. S. Mingatos \& M. Okumura. 2021. Taxonomic analysis of the Quaternary archaeofauna found at The Lapa do Santo site, Lagoa Santa region, Brazil. J. Quaternary Sci. 36: 12681278. DOI: https://doi.org/10.1002/ jqs. 3372

Chahud, A., G. S. Mingatos \& M. Okumura. 2020. Leporidae Fischer, 1817 (Mammalia: Lagomorpha) de um depósito Quaternário do Brasil: comentários taxonômicos e tafonômicos. Bol. Mus. Para. Goeldi. Ciências Naturais. 15(3): 795-806. DOI: http:// doi.org/10.46357/bcnaturais.v15i3.299

Chahud, A. \& M. Okumura. 2021a. The presence of Panthera onca Linnaeus 1758 (Felidae) in the Pleistocene of the region of Lagoa Santa, State of Minas Gerais, Brazil. Hist. Bio. 33(10): 2496-2503. DOI: https:// doi.org/10.1080/08912963.2020.1808975
Chahud, A. \& M. Okumura. 2021b. The youngest Tapir of a Quaternary deposit of the Americas. Hist. Bio. 33(10): 2400-2405. DOI: https://doi.org/10.1080/08912963.2020.1798420

Chahud, A. \& M. Okumura. 2022. Cervidae and Tayassuidae from the Holocene deposits of the Cuvieri Cave, State of Minas Gerais, eastern Brazil; taxonomic and paleoenvironmental considerations, Hist. Bio. DOI: https://doi.org/ 10.1080/08912963.2021.2022134

Emmons, L. 2016. Cuniculus paca. The IUCN Red List of Threatened Species 2016: e.T699A22197347. DOI: https://dx.doi.org/ 10.2305/IUCN.UK.2016-2.RLTS.T699A22197347.en.

Figuti, L. O. 1993. Homem pré-histórico, o molusco e o sambaqui: considerações sobre a subsistência dos povos sambaquieiros. Rev. Mus. Arqueol. Etnol. 3: 67-80.

Hingst-Zaher, E. \& M. V. Brandão. 2021. Atlas Craniano: mamíferos da Mata Atlântica e lista de espécies. 227 p.

Haddad-Martim, P. M., A. Hubbe, P. C. F. Giannini, A. S. Auler, L. B. Piló, M. Hubbe, E. Mayer, X.Wang, H. Cheng, R. L. Edwards, \& W. A. Neves. 2017. Quaternary depositional facies in cave entrances and their relation to landscape evolution: The example of Cuvieri Cave, eastern Brazil. Catena. 157: 372-387. DOI: https:// doi.org/10.1016/j.catena.2017.05.029

Hubbe, A., P. M. Haddad-Martim, M. Hubbe, E. L. Mayer, A. Strauss, A. S. Auler, L. B. Piló \& W. A. Neves. 2011. Identification and importance of critical depositional gaps in pitfall cave environments: the fossiliferous deposit of Cuvieri Cave, eastern Brazil. Palaeog, Palaeoc, Palaeoeco. 312: 66-78. DOI: https://doi.org/10.1016/j.palaeo.2011.09.010

Kerber, L. 2017. Imigrantes em um continente perdido: O registro fossilífero de roedores Caviomorpha (Mammalia: Rodentia: Ctenohystrica) do Cenozoico do Brasil. Terrae Didactica. 13(2): 185-211. DOI: http:// dx.doi.org/10.20396/td.v13i3.8650959

Leuchtenberger, C., F. Tirelli, F. Mazim, F. Peters, E. Oliveira, L. Cariolatto, D. Queirolo. 2017. New records of Cuniculus paca (Rodentia: Cuniculidae) in a temperate grassland dominated landscape of Pampa region of Brazil and Uruguay. Mammalia. 81. 425-428. DOI: https://doi.org/10.1515/mammalia-2015-0129 
Lund P. W. 1837. Om huler i kalksteen i det Indre af Brasilien, der tildeels indeholde fossile knokler. Anden Afhandling: Lappa da Cerca Grande (Forelaest, d. 2de Decbr. 1836). Det Kongelige Danske Videnskabernes Selskabs Naturvidenskabelige og Mathematiske Afhandlinger. 7: 307-332.

Lyman, R. L. 1994. Vertebrate Taphonomy, Cambridge Manuals in archaeology. Cambridge. $552 \mathrm{p}$.

Mayer E. L., A. Hubbe, L. Kerber, P. HaddadMartim \& W. Neves. 2016. Taxonomic, biogeographic, and taphonomic reassessment of a large extinct species of paca from the Pleistocene of Brazil. Acta Palaeo. Pol. 61(4): 743-758. DOI: https://doi.org/ 10.4202/app.00236.2016

Mayer, E. L. A., Hubbe, J. Botha-Brink, A. M. Ribeiro, P. M. Haddad-Martim \& W. Neves. 2020. Diagenetic changes on bone histology of Quaternary mammals from a tropical cave deposit in southeastern Brazil. Palaeog. Palaeoc. Palaeoeco. 537: 109372. DOI: https://doi.org/10.1016/j.palaeo.2019.109372

Mammal Diversity Database. 2021. Mammal diversity database (1.6). zenodo. DOI: http://doi.org/10.5281/zenodo.4139818

Mendes-Oliveira, A. C., S. L. de Maria, R. C. S. de Lima, A. S. Fernandes, P. C. de Almeida, L. F. Montag \& O. de Carvalho Jr. 2012. Testing simple criteria for de age estimation of six hunted mammal species in Brazilian Amazon. Mastozool. Neotrop. 19(1): 105-116.

Mingatos, G. S. \& M. Okumura. 2016. Modelo de amplitude de dieta aplicada a restos faunísticos do sítio Lapa do Santo (MG) e suas implicações para o entendimento da dieta em grupos Paleoíndios do Brasil central. Palaeoindian Archaeology. 1: 15-31.

Parizzi, M. G., M. L. Salgado-Labouriau \& H. C. Kholer. 1998. Genesis and environmental history of Lagoa Santa, southeastern Brazil. Holocene. 8: 311-321.
Patton, J. L., U. F. J. Pardiñas \& G. D'Elía. 2015. Mammals of South America. Rodents. Chicago, University of Chicago Press. 1336 p.

Paula Couto, C. 1979. Tratado de Paleomastozoologia. Rio de Janeiro, Academia Brasileira de Ciências. 590 p.

Pavei, D. D., J. B. Campos, J. J. Zocche \& M. C. P. Santos. 2015. Zooarqueologia de Vertebrados do Sambaqui do Papagaio, Bombinhas, Santa Catarina. Rev. Tec. e Amb. 21: 70-89

Plens, C. R. 2010. Animais para humanos na vida e na morte. Rev. Mus. Arqueol. Etnol. 20: 31-51. DOI: https://doi.org/10.11606/ issn.2448-1750.revmae.2010.89909

Ríos-Uzeda B., R. B. Wallace \& J. Vargas. 2004. La jayupa de la altura (Cuniculus taczanowskii, Rodentia, Cuniculidae), un nuevo registro de mamífero para la fauna de Bolivia, Mastozool. Neotrop. 11: 109-114.

Trolle, M., M. C. Bissaro \& H. M. Prado. 2006. Mammal survey at a ranch of the Brazilian Cerrado. pp. 379-385. In: Hawksworth, D. L. \& A. T. Bull (Eds.). Vertebrate Conservation and Biodiversity. Dordrecht, Springer. DOI: https://doi.org/10.1007/978-1-40206320-6 25

Voorhies, M. 1969. Taphonomy and population dynamics of an early Pliocene vertebrate fauna, Knox County, Nebraska. Contribution Geology Special Papers Univ. Wyo. Wyoming, Press Laramie. 69 p.

Woods, C. A. \& C. W. Kilpatrick. 2005. Infraorder Hystricognathi Brandt, 1855. pp. 1538-1600. In: Wilson, D. E. \& D. M. Reeder (Eds.). Mammal Species of the World: a taxonomic and geographic reference. 3. ed. Baltimore, The Johns Hopkins University Press.

\footnotetext{
Editor Cientifico / Scientific Editor: Sergio Solari, Universidad de Antioquia, Colômbia

Recebido / Recibido / Received: 11.07.2021

Revisado / Revised: 03.01.2022

Aceito / Aceptado / Accepted: 06.01.2022

Publicado / Published: 07.01.2022

DOI: https://doi.org/10.5216/rbn.v19i1.69286

Dados disponíveis / Datos disponibles / Available data: Repositório não informado
}

Rev. Biol. Neotrop. / J. Neotrop. Biol., Goiânia, v. 19, n. 1, p. 1-8, jan.-jun. 2022 